\section{IN THE NEWS}

Live by the sword

Whenever a scientific finding could become important for the improvement of human health, the press will quickly herald it as a breakthrough. But similarly, when things take a sour turn, they are hastily reported as major calamities. The first full-scale clinical trial of fetal-tissue transplantation to treat Parkinson's disease, the results of which appeared in the New England Journal of Medicine, is a good example. The study reported that none of 40 patients with Parkinson's disease who received sham or fetal cell transplants had noticed improvements in their condition. Moreover, most clinical assessments were also negative. One key finding was that $15 \%$ of patients who had received the fetal cells developed dyskinesias reminiscent of those induced by treatment with L-DOPA. So how did the news media report the story?

"Parkinson's miracle cure turns into a catastrophe." The Guardian (UK, 13 March)

The article then features quotes from Dr Paul Greene, one of the neurologists involved in the work.

Describing the side-effects, Dr Greene is quoted as saying: "It was tragic, catastrophic. And we can't selectively turn it off ... No more foetal transplants."

Robert Meadowcroft, director of policy and research at the Parkinson's Disease Society in the UK, said to The Independent (UK, 14 March) that the results were disappointing but that, in his view, they did not spell the end of any attempt to 'seed' the brain with either fetal cells, or in the future, stem cells.

Perhaps predictably, the heady mixture of the vexed ethical issue about the use of fetal transplants for medical treatments and the spectre of potentially irreversible sideeffects from a treatment previously hailed as a 'miracle cure' proved harder to resist for some newspapers.

Peter Collin

\title{
Monkey see, monkey do
}

One of the fastest ways to learn how to execute a given action is by watching someone else performing it first. Learning how to dance, how to play tennis and how to fix your tie are good examples of tasks that are best learned if we watch closely the movements of our teacher. How does the

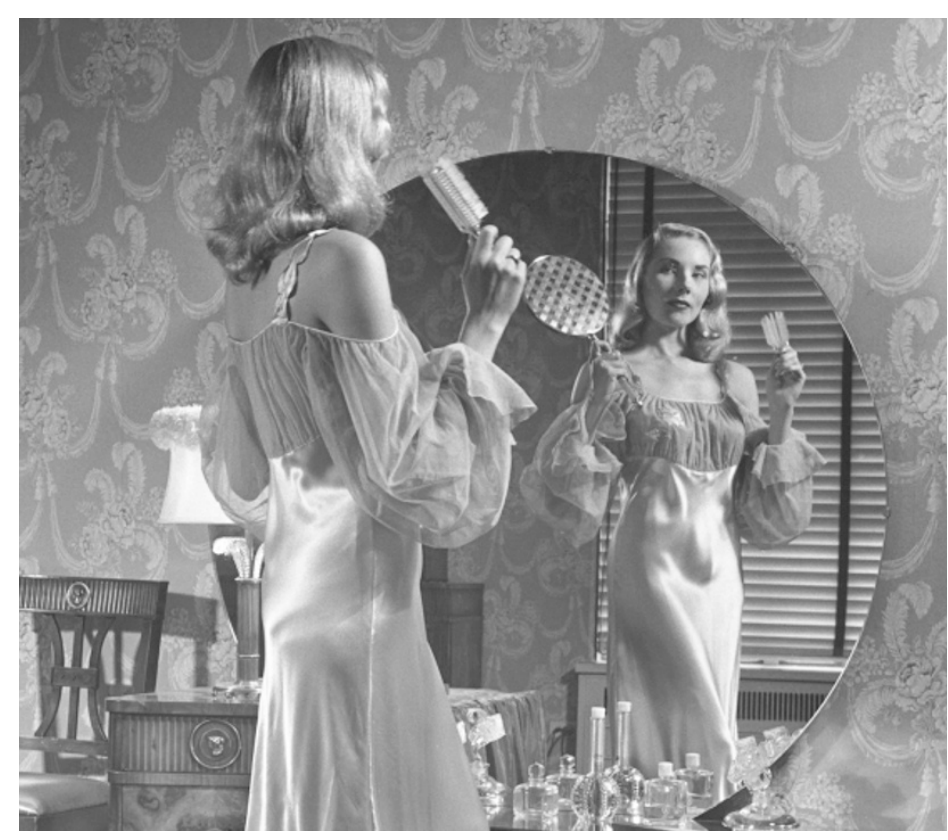

nervous system process someone else's actions? Is there something about the brain that makes it easier for us to imitate others? Only a few studies have addressed this question but a remarkable answer has begun to emerge from them: the primate brain possesses a 'mirror' system — neurons

LEARNING AND MEMORY

\section{Memories are made of this}

The human body is constantly producing new cells. Just think about how quickly new skin cells are produced to heal a cut, or the capacity of the bone marrow to produce blood cells. But for many years, neuroscientists believed that neurons in the human brain could not be renewed. Although some issues related to adult neurogenesis remain controversial, it has become increasingly clear that new neurons are constantly being produced even in the adult brain, particularly in the hippocampus. The well-known association of the hippocampus with memory and learning led naturally to speculation that these newborn neurons were involved in the formation of memories, and studies showed that associative learning could increase the number of newly formed cells that survived for long periods. But how could we test whether these cells are actually needed for learning? Tracey Shors and colleagues might have come up with the answer. Shors et al. took advantage of the ability of the DNA methylating agent methylazoxymethanol that are active both during the execution of an action and during the observation of the same action made by another subject. A recent study published in the European Journal of Neuroscience has obtained new evidence for the existence of a mirror system in the human brain by showing that, much like the motor system itself, the mirror system is organized in a somatotopic manner.

Buccino et al. carried out an imaging study in which subjects were required simply to watch clips of people performing motor actions with different parts of their bodies mouth, hand and foot. The authors found that watching the movements of another person led to the bilateral activation of the premotor cortex. Moreover, the pattern of activation resembled the motor homunculus watching someone chewing led to activation of the ventral premotor cortex, watching the movement of a foot elicited activation of the dorsal premotor area and watching someone move a hand caused the activation of an intermediate premotor region.

Another remarkable finding from this study was that the pattern of brain activation differed depending on whether the motor action involved the actual manipulation of an object. In other words, it was not the same to

(MAM) to kill proliferating cells. When the toxin was injected into adult rats, the number of new neurons produced in the dentate gyrus of the hippocampus was reduced by over $80 \%$. The team then tested whether these rats showed any learning impairment. They used two kinds of associative learning - delay conditioning, in which the conditioned and unconditioned stimuli overlap temporally, and trace conditioning, in which the two stimuli are separated in time. Crucially, trace conditioning is dependent on normal hippocampal function, whereas delay conditioning is not.

The researchers found that the rats performed normally in the delay conditioning task, but were 